\title{
Calculation of Thermal Pressure Coefficient of R11, R13, R14, R22, R23, R32, R41, and R113 Refrigerants by $p V T$ Data
}

\author{
Vahid Moeini and Mahin Farzad \\ Department of Chemistry, Payame Noor University, P.O. Box 19395-3697, Tehran, Iran \\ Correspondence should be addressed to Vahid Moeini; v_moeini@yahoo.com
}

Received 30 November 2012; Revised 2 March 2013; Accepted 10 May 2013

Academic Editor: Jan Skov Pedersen

Copyright (C) 2013 V. Moeini and M. Farzad. This is an open access article distributed under the Creative Commons Attribution License, which permits unrestricted use, distribution, and reproduction in any medium, provided the original work is properly cited.

For thermodynamic performance to be optimized particular attention must be paid to the fluid's thermal pressure coefficients and thermodynamic properties. A new analytical expression based on the statistical mechanics is derived for R11, R13, R14, R22, R23, $\mathrm{R} 32$, R41, and R113 refrigerants, using the intermolecular forces theory. In this paper, temperature dependency of the parameters of R11, R13, R14, R22, R23, R32, R41, and R113 refrigerants to calculate thermal pressure coefficients in the form of first order has been developed to second and third orders and their temperature derivatives of new parameters are used to calculate thermal pressure coefficients. These problems have led us to try to establish a function for the accurate calculation of the thermal pressure coefficients of R11, R13, R14, R22, R23, R32, R41, and R113 refrigerants based on statistical-mechanics theory for different refrigerants.

\section{Introduction}

Popular interest in the use of refrigerant blends started in the late 1950s. The emphasis was placed on energy savings through the reduction of irreversibility in the heat exchanger and on capacity variation during operation through the control of the fluid composition. Worldwide legislation has been enacted through the United Nations environmental program to reduce stratospheric ozone depletion. The Montreal Protocol was approved in 1987 to control production of the suspected ozone-depleting substances, among them chlorofluorocarbons and hydrochlorofluorocarbons, commonly used as refrigerants in the industry. For example, chlorofluorocarbons-(CFCs-) 11, 12 and 113 have been successfully used to determine groundwater recharge ages in the industry. Relatively good agreement exists between individual CFC ages and ages derived from other tracers [1-6].

The precise meaning of the internal pressure is contained in a generalized manner in the following well-known thermodynamic equations. United forces of external and internal pressure equalize the thermal pressure which tries to expand the matter. If the thermal pressure of a refrigerant is available, then the thermodynamics properties of refrigerant can be calculated easily. Liquids and dense fluids are usually considered to be complicated on a molecular scale, and a satisfactory theory of liquids only began to emerge in the 1960. However, they show a number of experimental regularities, some of which have been known by theoretical basis [7-10].

The first is the internal pressure regularity, in which $\left((\partial E / \partial V)_{T} / \rho R T\right) V^{2}$ is linear with respect to $\rho^{2}$ for each isotherm, where $\rho=1 / V$ is the molar density, $E$ is the internal energy, and $V$ is molar volume [9]. In the internal pressure regularity, which was originally devised for normal dense fluids, is based on the cell theory and considers only nearest adjacent interaction. Lennard-Jones potential function suitably describes the interactions between the molecules of a fluid under the condition that it behaves as a normal fluid. In the internal pressure regularity was attempted to calculate the internal pressure by modeling the average configurationally potential energy and then taking its derivative with respect to volume.

The second is an expression which is driven for as the thermal pressure coefficient of dense fluids $\left(\mathrm{Ar}, \mathrm{N}_{2}, \mathrm{CO}\right.$, $\mathrm{CH}_{4}, \mathrm{C}_{2} \mathrm{H}_{6}, \mathrm{n}-\mathrm{C}_{4} \mathrm{H}_{10}$, iso- $\mathrm{C}_{4} \mathrm{H}_{10}, \mathrm{C}_{6} \mathrm{H}_{6}$, and $\mathrm{C}_{6} \mathrm{H}_{5}-\mathrm{CH}_{3}$ ) [1117]. Only, $p V T$ experimental data have been used for the calculation of thermal pressure coefficient [18].

The third is a regularity to predict metal-nonmetal transitions in cesium fluid. An accurate empirical potential has 
been found for dense cesium fluid and it is used to test the applicability of the theory. These theoretical predictions are in good agreement with experimental results [19-26].

The forth is the internal pressure of sodium, potassium, and rubidium attempt to predict X-ray diffraction and smallangle X-ray scattering to the range where the compressibility of the interacting electron gas has been theoretically predicted to become negative. Problems have led us to try to establish a function for the accurate calculation of the internal pressure and the prediction of metal-nonmetal transition alkali metals based on the internal pressure [27].

A property formulation is the set of equations used to calculate properties of a fluid at specified thermodynamic states defined by an appropriate number of independent variables. A typical thermodynamic property formulation is based on an equation of state which allows the correlation and computation of all thermodynamic properties of the fluid including properties such as entropy that cannot be measured directly. Modern equations of state at least with 17 terms for pure fluid properties are usually fundamental equations explicit in the Helmholtz energy as a function of density and temperature. The new class of equations of state for technical applications to dense fluids is formulated in the reduced Helmholtz energy. As usual, the reduced Helmholtz energy is split into one part which describes the behavior of the hypothetical ideal gas at given values of temperature and density and a second part which describes the residual behavior of the fluid. For some relevant properties the corresponding relations were given in $[4,9]$.

In 1993 a general regularity with 2 terms so-called the linear isotherm regularity has been reported for pure dense fluids, according to which $(Z-1) V^{2}$ is linear with respect to $\rho^{2}$, each isotherm as

$$
(Z-1) V^{2}=A+B \rho^{2}
$$

where $Z \equiv p V / R T$ is the compression factor, $\rho=1 / V$ is the molar density, and $A$ and $B$ are the temperaturedependent parameters. This equation of state works very well for all types of dense fluids, for densities greater than the Boyle density but for temperatures below twice the Boyle temperature. The regularity was originally suggested on the basis of a simple lattice-type model applied to a Lennard-Jones $(12,6)$ fluid $[28,29]$. At present work, the regularity has been used to calculate thermal pressure coefficient of dense Trichlorofluoromethane (R11), Chlorotrifluoromethane (R13), Tetrafluoromethane (R14), Chlorodifluoromethane (R22), Trifluoromethane (R23), Difluoromethane (R32), Fluoromethane (R41), and 1,1,2Trichloro-1,2,2-trifluoroethane (R113) refrigerants [30]. In this paper, in Section 2.1, we present a simple method that keeps first order temperature dependency of parameters in the regularity versus inverse temperature. Then, the thermal pressure coefficient is calculated by this expression. In Section 2.2, temperature dependency of parameters in the regularity has been developed to second order. In Section 2.3, temperature dependency of parameters in the regularity has been developed to third order and then the thermal pressure coefficient is calculated in each state for R11, R13, R14, R22, R23, R32, R41, and R113 refrigerants.

\section{Theory}

We fist test the ability of the linear isotherm regularity [18]

$$
\frac{p}{\rho R T}=1+A \rho^{2}+B \rho^{4} .
$$

2.1. First Order Temperature Dependency of Parameters. We first calculate pressure by the linear isotherm regularity and then use first order temperature dependency of parameters to get the thermal pressure coefficient for the dense fluid, where

$$
\begin{aligned}
& A=A_{2}-\frac{A_{1}}{R T}, \\
& B=\frac{B_{1}}{R T} .
\end{aligned}
$$

Here $A_{1}$ and $B_{1}$ are related to the intermolecular attractive and repulsive forces, respectively, while $A_{2}$ is related to the nonideal thermal pressure and $R T$ has its usual meaning.

In the present work, the starting point in the derivation is (2). By substitution of (3) and (4) in (2) we obtain the pressure for R11, R13, R14, R22, R23, R32, R41, and R113 fluids:

$$
p=\rho R T+A_{2} \rho^{3} R T-A_{1} \rho^{3}+B_{1} \rho^{5} .
$$

We first drive an expression for thermal pressure coefficient using first order temperature dependency of parameters. The final result is $\mathrm{TPC}^{(1)}$ :

$$
\left(\frac{\partial p}{\partial T}\right)_{\rho}=R \rho+A_{2} R \rho^{3} .
$$

According to (6), the experimental value of density and value of $A_{2}$ from Table 1 can be used to calculate the value of thermal pressure coefficient.

For this purpose we have plotted $A$ versus $1 / T$ whose intercept shows value of $A_{2}$. Table 1 shows the $A_{2}$ values for R11, R13, R14, R22, R23, R32, R41, and R113 fluids [1, 6, 30].

Then we obtain the thermal pressure coefficient of dense fluids. R13 serves as our primary test fluid because of the abundance of available thermal pressure coefficients data $[6,30]$. For this purpose we have plotted $A$ versus $1 / T$ whose intercept shows value of $A_{2}$. Figures 1(a) and 1(b) show plots of $A$ and $B$ versus inverse temperature for R13, respectively. It is clear that $A$ and $B$ versus inverse temperature are not first order.

2.2. Second Order Temperature Dependency of Parameters. In order to solve this problem, the linear isotherm regularity equation of state in the form of truncated temperature series of $A$ and $B$ parameters has been developed to second order for dense fluids. Figures 1(a) and 1(b) show plots of $A$ and $B$ parameters versus inverse temperature for R13 fluid. It is clear that $A$ and $B$ versus inverse temperature are second order. 


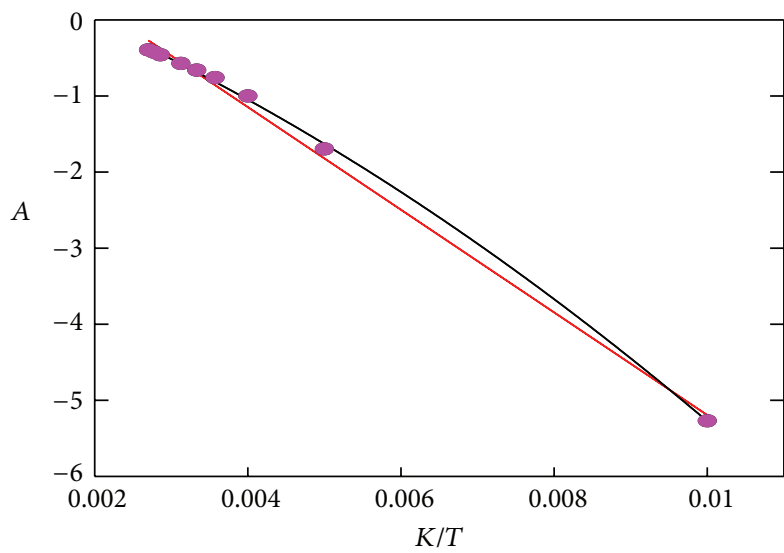

(a)

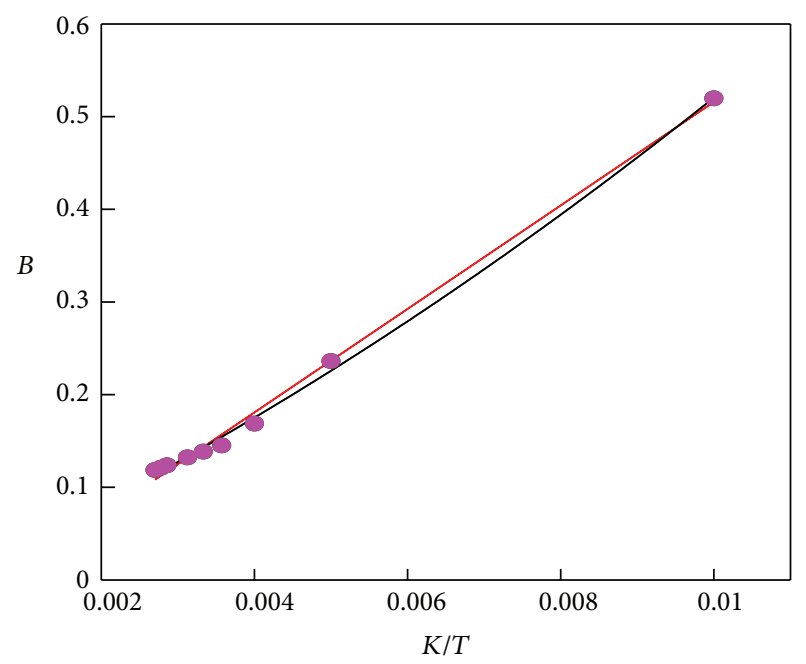

(b)

Figure 1: (a) Plot of $A$ versus inverse temperature. Red and blue lines are the first and second order fit to the $A$ data points, for R13, respectively. (b) Plot of $B$ versus inverse temperature. Red and blue lines are the first and second order fit to the $B$ data points, for R13, respectively.

TABLE 1: The calculated values of $A_{2}$ for different fluids using (3) and the coefficient of determination $\left(R^{2}\right)[1,6,18,30]$.

\begin{tabular}{lccc}
\hline Fluid & $A_{2}$ & $\left(T_{\min }-T_{\max }\right) / \mathrm{K}$ & $R^{2}$ \\
\hline R11 & 2.2109 & $180-500$ & 0.9849 \\
R13 & 1.5748 & $100-370$ & 0.9946 \\
R14 & 1.1315 & $150-310$ & 0.9894 \\
R22 & 1.5616 & $120-500$ & 0.9890 \\
R23 & 1.0286 & $150-450$ & 0.9905 \\
R32 & 0.8650 & $150-430$ & 0.9909 \\
R41 & 0.6263 & $180-410$ & 0.9927 \\
R113 & 1.3863 & $300-525$ & 0.9977 \\
\hline
\end{tabular}

Thus, we obtain extending parameters $A$ and $B$ resulting in second order equation as follows:

$$
\begin{aligned}
& A=A_{1}+\frac{A_{2}}{T}+\frac{A_{3}}{T^{2}}, \\
& B=B_{1}+\frac{B_{2}}{T}+\frac{B_{3}}{T^{2}} .
\end{aligned}
$$

The starting point in the derivation is (2) again. By substitution of (7)-(8) in equation (2) we obtain the pressure for R11, R13, R14, R22, R23, R32, R41, and R113 fluids [1, 6, 30]:

$$
\begin{aligned}
p= & \rho R T+A_{1} \rho^{3} R T+A_{2} \rho^{3} R \\
& +\frac{A_{3} \rho^{3} R}{T}+B_{1} \rho^{5} R T+B_{2} \rho^{5} R+\frac{B_{3} \rho^{5} R}{T} .
\end{aligned}
$$

First, second, and third temperature coefficients and their temperature derivatives were calculated from this model and
TABLE 2: The calculated values of $A_{1}, A_{3}$ using (7) and $B_{1}, B_{3}$ using (8) for different fluids and the coefficient of determination $\left(R^{2}\right)[1,6$, $18,30]$.

\begin{tabular}{lcccccc}
\hline Fluid & $A_{1}$ & $A_{3}$ & $R^{2}$ & $B_{1}$ & $B_{3}$ & $R^{2}$ \\
\hline R11 & 0.1354 & $-1.7517 \times 10^{5}$ & 0.9987 & 0.0625 & $1.4777 \times 10^{4}$ & 0.9997 \\
R13 & 1.3594 & -7165.5660 & 0.9898 & -0.0650 & -610.8607 & 0.9951 \\
R14 & 0.1810 & $-4.2972 \times 10^{4}$ & 0.9992 & 0.1072 & 5461.0737 & 0.9997 \\
R22 & 0.6210 & $-4.8725 \times 10^{4}$ & 0.9983 & 0.0196 & 3298.4237 & 0.9994 \\
R23 & 0.4457 & $-3.4069 \times 10^{4}$ & 0.9993 & 0.0317 & 2449.0303 & 0.9997 \\
R32 & 0.2404 & $-3.8638 \times 10^{4}$ & 0.9980 & 0.0528 & 3251.8493 & 0.9996 \\
R41 & 0.0924 & $-3.7820 \times 10^{4}$ & 0.9982 & 0.0850 & 4669.1360 & 0.9996 \\
R113 & 0.6053 & $-1.2415 \times 10^{5}$ & 0.9992 & 0.0316 & 7746.1673 & 0.9997 \\
\hline
\end{tabular}

the final result is for the thermal pressure coefficient to form $\mathrm{TPC}^{(2)}$ :

$$
\left(\frac{\partial p}{\partial T}\right)_{\rho}=\rho R+A_{1} \rho^{3} R-\frac{A_{3} \rho^{3} R}{T^{2}}+B_{1} \rho^{5} R-\frac{B_{3} \rho^{5} R}{T^{2}} .
$$

As (10) shows, it is possible to calculate the thermal pressure coefficient at each density and temperature by knowing $A_{1}, A_{3}, B_{1}, B_{3}$. For this purpose we have plotted extending parameters of $A$ and $B$ versus $1 / T$ whose intercept and coefficients show the values of $A_{1}, A_{3}, B_{1}, B_{3}$ that are given in Table 2.

2.3. Third Order Temperature Dependency of Parameters. In another step, we test to form truncated temperature series of $A$ and $B$ parameters to third order:

$$
\begin{gathered}
A=A_{1}+\frac{A_{2}}{T}+\frac{A_{3}}{T^{2}}+\frac{A_{4}}{T^{3}}, \\
B=B_{1}+\frac{B_{2}}{T}+\frac{B_{3}}{T^{2}}+\frac{B_{4}}{T^{3}} .
\end{gathered}
$$


TABLE 3: The calculated values of $A_{1}, A_{3}, A_{4}$ using (11) and $B_{1}, B_{3}, B_{4}$ using (12) for different fluids and the coefficient of determination ( $\left.R^{2}\right)$ $[1,6,18,30]$.

\begin{tabular}{lccccccrc}
\hline Fluid & $A_{1}$ & $A_{3}$ & $A_{4}$ & $R^{2}$ & $B_{1}$ & $B_{3}$ & $B_{4}$ \\
\hline R11 & -1.1003 & $-5.2851 \times 10^{5}$ & $3.2477 \times 10^{7}$ & 0.9999 & 0.3293 & $9.1064 \times 10^{4}$ & $-7.0120 \times 10^{5}$ & 0.9999 \\
R13 & -1.2784 & $-2.9997 \times 10^{5}$ & $1.5854 \times 10^{7}$ & 0.9999 & 0.2582 & $9.5263 \times 10^{4}$ & $-1.9424 \times 10^{6}$ & 0.9999 \\
R14 & 0.8257 & $4.5033 \times 10^{4}$ & $-5.9456 \times 10^{6}$ & 0.9998 & 0.0642 & -402.0298 & $3.6911 \times 10^{5}$ & 0.9993 \\
R22 & $8.1 \times 10^{-3}$ & $-1.5322 \times 10^{5}$ & $6.9130 \times 10^{6}$ & 0.9999 & 0.0988 & $1.6789 \times 10^{4}$ & $-8.9251 \times 10^{5}$ & 0.9998 \\
R23 & 0.2352 & $-7.5616 \times 10^{4}$ & $3.1780 \times 10^{6}$ & 0.9998 & 0.0618 & 8402.7408 & $-4.5542 \times 10^{5}$ & 0.9993 \\
R32 & -0.1924 & $-1.2103 \times 10^{5}$ & $6.2039 \times 10^{6}$ & 0.9999 & 0.1290 & $1.7760 \times 10^{4}$ & $-1.0925 \times 10^{6}$ & 0.9995 \\
R41 & 0.6315 & $8.2985 \times 10^{4}$ & $-1.0303 \times 10^{7}$ & 0.9999 & 0.0298 & -7705.9129 & $1.0555 \times 10^{6}$ & 0.9988 \\
R113 & -0.9445 & $-8.5187 \times 10^{5}$ & $9.2993 \times 10^{7}$ & 0.9998 & 0.2258 & $9.8942 \times 10^{4}$ & $-1.1654 \times 10^{7}$ & 0.9995 \\
\hline
\end{tabular}

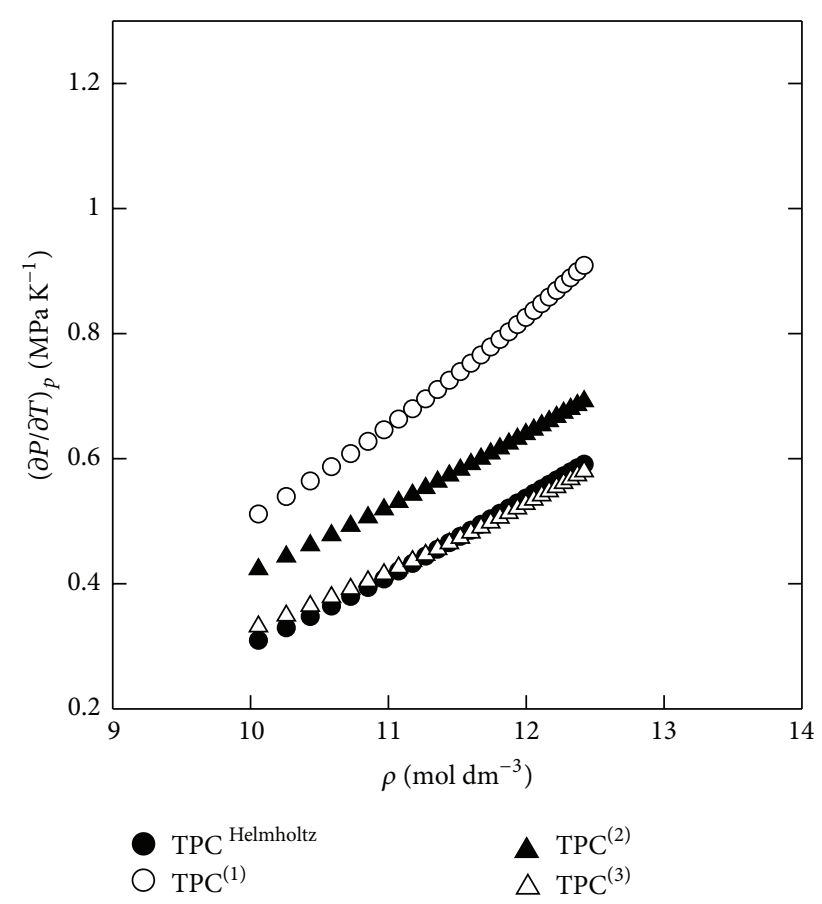

FIgURE 2: The experimental values of thermal pressure coefficient versus density for R13 fluid are compared with thermal pressure coefficient using the $\mathrm{TPC}^{(1)}, \mathrm{TPC}^{(2)}$, and $\mathrm{TPC}^{(3)}$ at $300 \mathrm{~K}$.

The starting point in the derivation is (2) again. By substitution of (11) and (12) in (2) we obtain the pressure equation for R11, R13, R14, R22, R23, R32, R41, and R113 fluids [1, 6, 30]:

$$
\begin{aligned}
p= & \rho R T+A_{1} \rho^{3} R T+A_{2} \rho^{3} R+\frac{A_{3} \rho^{3} R}{T}+\frac{A_{4} \rho^{3} R}{T^{2}} \\
& +B_{1} \rho^{5} R T+B_{2} \rho^{5} R+\frac{B_{3} \rho^{5} R}{T}+\frac{B_{4} \rho^{5} R}{T^{2}} .
\end{aligned}
$$

The final result is for the thermal pressure coefficient to form $\mathrm{TPC}^{(3)}$ :

$$
\begin{aligned}
\left(\frac{\partial p}{\partial T}\right)_{\rho}= & \rho R+A_{1} \rho^{3} R-\frac{A_{3} \rho^{3} R}{T^{2}}-\frac{2 A_{4} \rho^{3} R}{T^{3}} \\
& +B_{1} \rho^{5} R-\frac{B_{3} \rho^{5} R}{T^{2}}-\frac{2 B_{4} \rho^{5} R}{T^{3}} .
\end{aligned}
$$

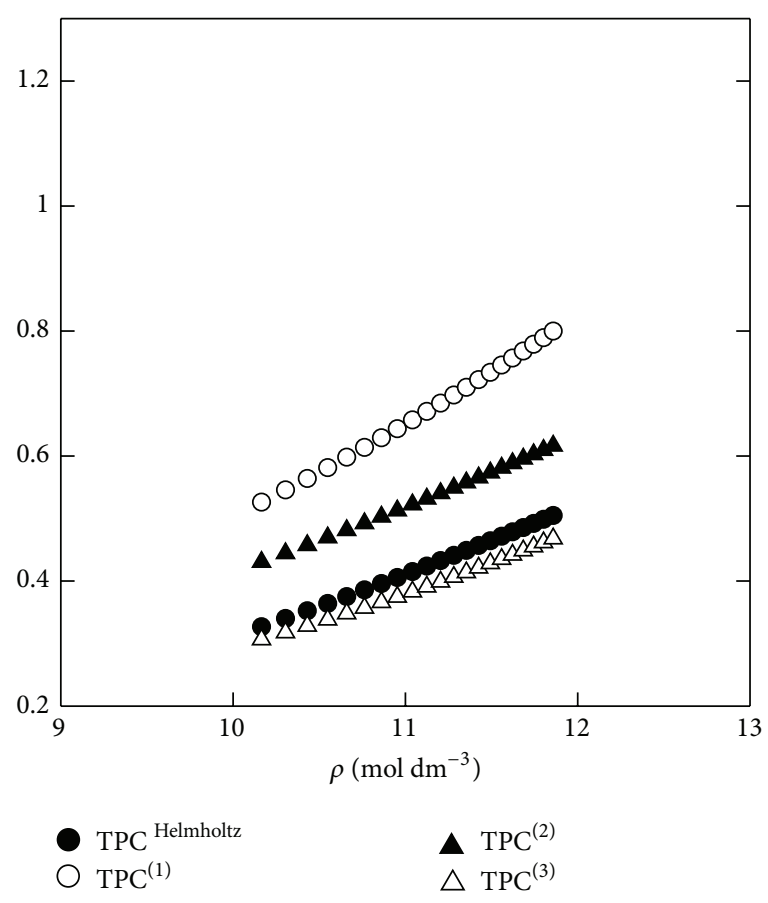

FIGURE 3: The experimental values of thermal pressure coefficient versus density for R13 fluid are compared with thermal pressure coefficient using the $\mathrm{TPC}^{(1)}, \mathrm{TPC}^{(2)}$, and $\mathrm{TPC}^{(3)}$ at $320 \mathrm{~K}$.

Based on (14), to obtain the thermal pressure coefficient, it is necessary to determine values $A_{1}, A_{3}, A_{4}, B_{1}, B_{3}, B_{4}$ these values are given in Table 3 . In contrast, Figures 2 and 3 show the experimental values of the thermal pressure coefficient versus density for R13 of liquid and supercritical fluids that are compared with the thermal pressure coefficient using the $\mathrm{TPC}^{(1)}, \mathrm{TPC}^{(2)}$, and $\mathrm{TPC}^{(3)}$ at 300 and $320 \mathrm{~K}$, respectively.

\section{Experimental Tests and Discussion}

A compromised ozone layer results in increased ultraviolet (UV) radiation reaching the earth's surface, which can have wide ranging health effects. Global climate change is believed to be caused by buildup of greenhouse gases in the atmosphere. The primary greenhouse gas is carbon dioxide $\left(\mathrm{CO}_{2}\right)$, created by fossil fuel-burning power plants. 


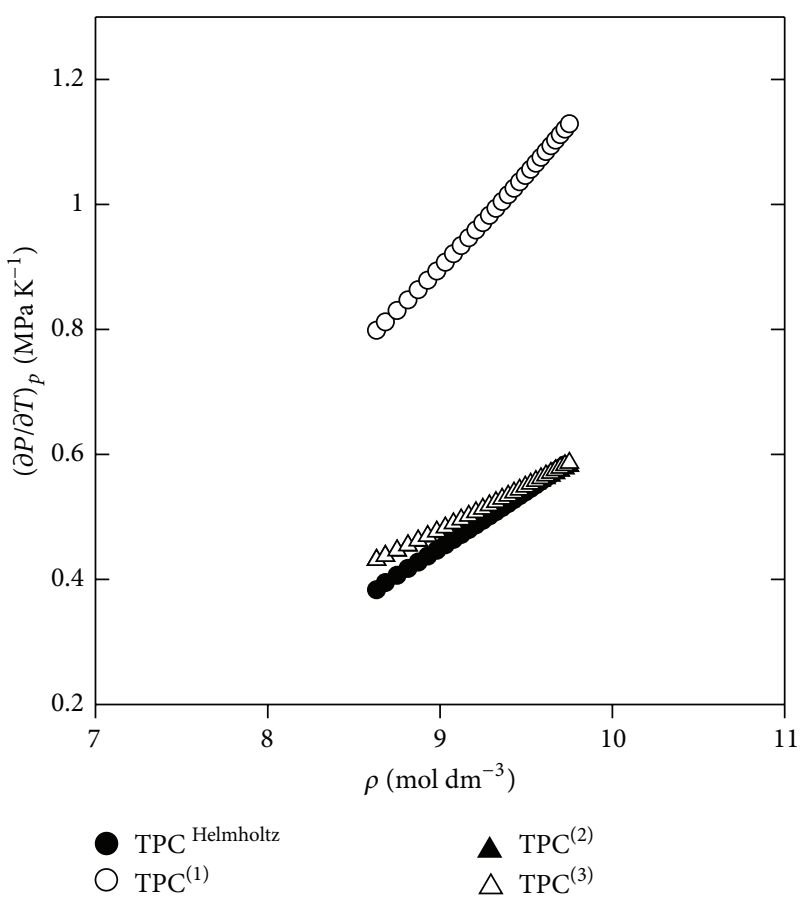

FIgURE 4: The experimental values of thermal pressure coefficient versus density for R11 fluid are compared with thermal pressure coefficient using the $\mathrm{TPC}^{(1)}, \mathrm{TPC}^{(2)}$, and $\mathrm{TPC}^{(3)}$ at $400 \mathrm{~K}$.

These gases trap the earth's heat, causing global warming. CFC, HCFC, and HFC refrigerants are considered greenhouse gases. The accurate description of thermodynamic properties of fluids over the large intervals of temperatures and densities with multiparameter equations of state has been a subject of active research, which has developed continuously during the last 30 years and will continue to do so. Generally, three categories of equations of state can be established according to their fundamentals: empirical, theoretical, and semiempirical. An empirical equation of state is usually needed to several experimental data or many adjustable parameters and therefore their applications are usually restricted to a very limited number of substances. A theoretical equation of state is also needed to the same number of molecular parameters, particularly to the intermolecular pair potential function $[1-6$, 30].

In this work, the thermal pressure coefficient is computed for refrigerant fluids of liquid and super critical fluids using three different models of the theoretical equation of state. R13 serves as our primary test fluid because of the abundance of available thermal pressure coefficients data. Such data are more limited for the other fluids examined. When we restricted temperature series of $A$ and $B$ parameters to first order it has been seen that the points from the low densities for $\mathrm{TPC}^{(1)}$ deviate significantly from the experimental data. To decrease adequately deviation the thermal pressure coefficient from the experimental data, it was necessary to extend the temperature series of $A$ and $B$ parameters to second order. The present approach to

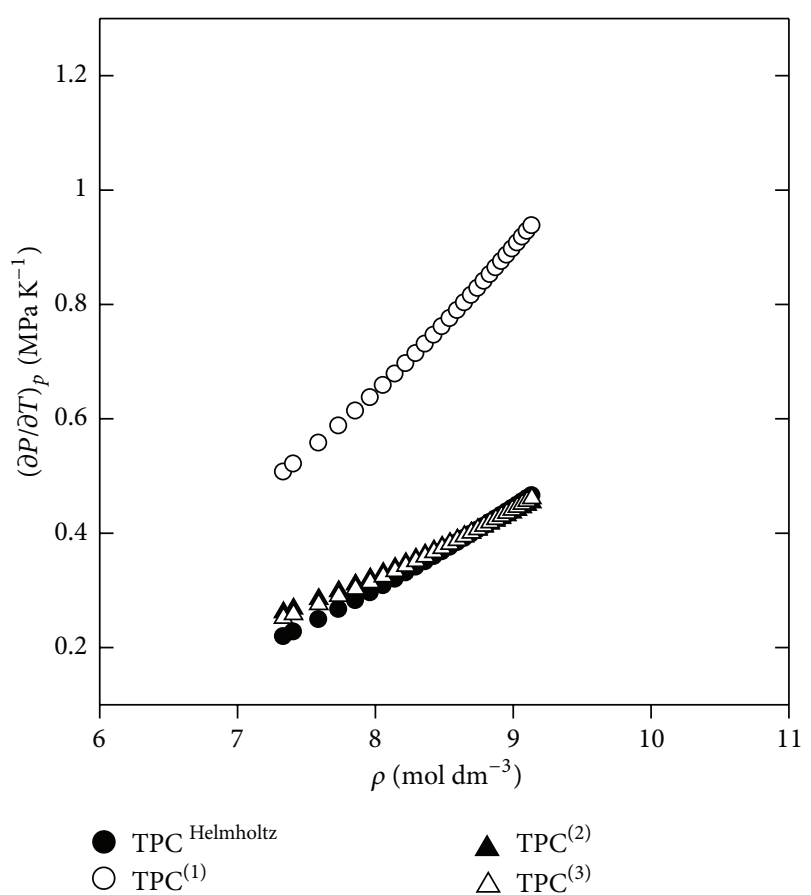

FIGURE 5: The experimental values of thermal pressure coefficient versus density for R11 fluid are compared with thermal pressure coefficient using the $\mathrm{TPC}^{(1)}, \mathrm{TPC}^{(2)}$, and $\mathrm{TPC}^{(3)}$ at $420 \mathrm{~K}$.

TABLE 4: Comparison of $(\partial p / \partial T)_{\rho}$ among the calculated and experimental values for R11 at $400 \mathrm{~K}[1,18,30]$.

\begin{tabular}{lccccc}
\hline \multirow{2}{*}{$p / \mathrm{MPa}$} & $\rho / \mathrm{mol} \cdot \mathrm{L}^{-1}$ & \multicolumn{4}{c}{$(\partial p / \partial T)_{\rho}\left(\mathrm{MPa} \cdot \mathrm{K}^{-1}\right)$} \\
& & $\mathrm{TPC}^{\mathrm{Helmholtz}}$ & $\mathrm{TPC}^{(1)}$ & $\mathrm{TPC}^{(2)}$ & $\mathrm{TPC}^{(3)}$ \\
\hline 1.4074 & 8.6318 & 0.3834 & 0.7986 & 0.4312 & 0.4310 \\
2.1000 & 8.6827 & 0.3948 & 0.8120 & 0.4375 & 0.4375 \\
3.1000 & 8.7509 & 0.4065 & 0.8301 & 0.4460 & 0.4462 \\
4.1000 & 8.8142 & 0.4174 & 0.8472 & 0.4539 & 0.4544 \\
5.1000 & 8.8732 & 0.4276 & 0.8633 & 0.4614 & 0.4621 \\
6.1000 & 8.9286 & 0.4373 & 0.8787 & 0.4685 & 0.4694 \\
7.1000 & 8.9810 & 0.4465 & 0.8933 & 0.4753 & 0.4764 \\
8.1000 & 9.0306 & 0.4554 & 0.9074 & 0.4818 & 0.4831 \\
9.1000 & 9.0780 & 0.4638 & 0.9210 & 0.4880 & 0.4895 \\
10.1000 & 9.1232 & 0.4719 & 0.9340 & 0.4940 & 0.4957 \\
11.1000 & 9.1665 & 0.4797 & 0.9467 & 0.4998 & 0.5017 \\
12.1000 & 9.2081 & 0.4872 & 0.9589 & 0.5053 & 0.5075 \\
13.1000 & 9.2481 & 0.4945 & 0.9708 & 0.5107 & 0.5131 \\
14.1000 & 9.2868 & 0.5015 & 0.9824 & 0.5160 & 0.5185 \\
15.1000 & 9.3241 & 0.5083 & 0.9937 & 0.5211 & 0.5238 \\
16.1000 & 9.3602 & 0.5149 & 1.0046 & 0.5260 & 0.5289 \\
17.1000 & 9.3952 & 0.5214 & 1.0154 & 0.5309 & 0.5339 \\
18.1000 & 9.4292 & 0.5277 & 1.0259 & 0.5356 & 0.5388 \\
19.1000 & 9.4623 & 0.5338 & 1.0361 & 0.5402 & 0.5436 \\
20.1000 & 9.4944 & 0.5398 & 1.0462 & 0.5447 & 0.5483 \\
21.1000 & 9.5257 & 0.5456 & 1.0560 & 0.5491 & 0.5529 \\
22.1000 & 9.5562 & 0.5513 & 1.0657 & 0.5534 & 0.5574 \\
23.1000 & 9.5859 & 0.5569 & 1.0752 & 0.5576 & 0.5618 \\
24.1000 & 9.6150 & 0.5624 & 1.0845 & 0.5617 & 0.5661 \\
25.1000 & 9.6434 & 0.5678 & 1.0937 & 0.5658 & 0.5703 \\
\hline & & & & &
\end{tabular}


TABLE 5: Comparison of $(\partial p / \partial T)_{\rho}$ among the calculated and experimental values for R11 at $440 \mathrm{~K}[1,18,30]$.

\begin{tabular}{lccccc}
\hline \multirow{2}{*}{$p / \mathrm{MPa}$} & $\rho / \mathrm{mol}^{-} \mathrm{L}^{-1}$ & \multicolumn{4}{c}{$(\partial p / \partial T)_{\rho}\left(\mathrm{MPa} \cdot \mathrm{K}^{-1}\right)$} \\
& & $\mathrm{TPC}^{\text {Helmholtz }}$ & $\mathrm{TPC}^{(1)}$ & $\mathrm{TPC}^{(2)}$ & $\mathrm{TPC}^{(3)}$ \\
\hline 2.7745 & 7.3336 & 0.2189 & 0.5067 & 0.2614 & 0.2515 \\
3.1000 & 7.4066 & 0.2271 & 0.5208 & 0.2679 & 0.2581 \\
4.1000 & 7.5898 & 0.2486 & 0.5572 & 0.2846 & 0.2755 \\
5.1000 & 7.7349 & 0.2664 & 0.5873 & 0.2983 & 0.2899 \\
6.1000 & 7.8569 & 0.2818 & 0.6135 & 0.3102 & 0.3024 \\
7.1000 & 7.9630 & 0.2955 & 0.6369 & 0.3207 & 0.3137 \\
8.1000 & 8.0576 & 0.3080 & 0.6582 & 0.3304 & 0.3240 \\
9.1000 & 8.1431 & 0.3195 & 0.6779 & 0.3392 & 0.3335 \\
10.1000 & 8.2215 & 0.3302 & 0.6964 & 0.3475 & 0.3424 \\
11.1000 & 8.2941 & 0.3403 & 0.7138 & 0.3552 & 0.3509 \\
12.1000 & 8.3617 & 0.3498 & 0.7302 & 0.3626 & 0.3589 \\
13.1000 & 8.4251 & 0.3587 & 0.7459 & 0.3695 & 0.3665 \\
14.1000 & 8.4850 & 0.3673 & 0.7609 & 0.3762 & 0.3738 \\
15.1000 & 8.5417 & 0.3755 & 0.7753 & 0.3826 & 0.3808 \\
16.1000 & 8.5956 & 0.3833 & 0.7892 & 0.3887 & 0.3876 \\
17.1000 & 8.6470 & 0.3909 & 0.8026 & 0.3946 & 0.3942 \\
18.1000 & 8.6962 & 0.3981 & 0.8155 & 0.4003 & 0.4005 \\
19.1000 & 8.7434 & 0.4052 & 0.8281 & 0.4058 & 0.4067 \\
20.1000 & 8.7887 & 0.4120 & 0.8403 & 0.4112 & 0.4127 \\
21.1000 & 8.8324 & 0.4185 & 0.8521 & 0.4164 & 0.4186 \\
22.1000 & 8.8746 & 0.4249 & 0.8637 & 0.4215 & 0.4243 \\
23.1000 & 8.9153 & 0.4312 & 0.8750 & 0.4264 & 0.4298 \\
24.1000 & 8.9547 & 0.4372 & 0.8860 & 0.4312 & 0.4353 \\
25.1000 & 8.9930 & 0.4431 & 0.8967 & 0.4359 & 0.4406 \\
26.1000 & 9.0301 & 0.4489 & 0.9073 & 0.4404 & 0.4458 \\
\hline & & & & & \\
& & & &
\end{tabular}

obtaining the thermal pressure coefficient from $p V T$ data contrasts with the experimental data by extending the temperature series of $A$ and $B$ parameters to second order and its derivatives. That, the thermal pressure coefficient give to form $\mathrm{TPC}^{(2)}$.

We also considered an even more accurate estimates namely, the extension of temperature series of $A$ and $B$ parameters to third order. The final result is for the thermal pressure coefficient to form $\mathrm{TPC}^{(3)}$. In contrast, Figures 4 and 5 show the experimental values of the thermal pressure coefficient versus density for R11 of liquid fluid that are compared with the thermal pressure coefficient using the $\mathrm{TPC}^{(1)}, \mathrm{TPC}^{(2)}$, and $\mathrm{TPC}^{(3)}$ at 400 and $440 \mathrm{~K}$, respectively. Also, the experimental and calculated values of the thermal pressure coefficient using the $\mathrm{TPC}^{(1)}, \mathrm{TPC}^{(2)}$, and $\mathrm{TPC}^{(3)}$ are compared in Tables 4, 5, 6, and 7 for R13 and R11 fluids. Although all three models capture the qualitative features for refrigerants, the calculated values of the thermal pressure coefficient using the $\mathrm{TPC}^{(2)}$ model produce quantitative agreement, but Tables $4,5,6$, and 7 , which is a more test of these models, shows that the $\mathrm{TPC}^{(3)}$ model is able to accurately predict both the thermal pressure coefficient of the liquid and the supercritical refrigerants.
TABLE 6: Comparison of $(\partial p / \partial T)_{\rho}$ among the calculated and experimental values for R13 at $300 \mathrm{~K}[1,6,18,30]$.

\begin{tabular}{lccccc}
\hline \multirow{2}{*}{$p / \mathrm{MPa}$} & $\rho / \mathrm{mol} \cdot \mathrm{L}^{-1}$ & \multicolumn{4}{c}{$(\partial p / \partial T)_{\rho}\left(\mathrm{MPa} \cdot \mathrm{K}^{-1}\right)$} \\
& & $\mathrm{TPC}^{\mathrm{Helmholtz}}$ & $\mathrm{TPC}^{(1)}$ & $\mathrm{TPC}^{(2)}$ & $\mathrm{TPC}^{(3)}$ \\
\hline 8.0000 & 10.0570 & 0.3095 & 0.5114 & 0.4231 & 0.3317 \\
9.0000 & 10.2590 & 0.3295 & 0.5393 & 0.4434 & 0.3490 \\
10.0000 & 10.4330 & 0.3476 & 0.5643 & 0.4614 & 0.3645 \\
11.0000 & 10.5870 & 0.3642 & 0.5870 & 0.4776 & 0.3786 \\
12.0000 & 10.7260 & 0.3796 & 0.6081 & 0.4925 & 0.3917 \\
13.0000 & 10.8520 & 0.3940 & 0.6276 & 0.5061 & 0.4038 \\
14.0000 & 10.9680 & 0.4075 & 0.6460 & 0.5189 & 0.4152 \\
15.0000 & 11.0750 & 0.4203 & 0.6633 & 0.5309 & 0.4260 \\
16.0000 & 11.1750 & 0.4324 & 0.6797 & 0.5421 & 0.4362 \\
17.0000 & 11.2700 & 0.4440 & 0.6956 & 0.5530 & 0.4461 \\
18.0000 & 11.3580 & 0.4550 & 0.7106 & 0.5631 & 0.4554 \\
19.0000 & 11.4430 & 0.4656 & 0.7252 & 0.5729 & 0.4645 \\
20.0000 & 11.5230 & 0.4757 & 0.7392 & 0.5823 & 0.4732 \\
21.0000 & 11.5990 & 0.4854 & 0.7526 & 0.5912 & 0.4816 \\
22.0000 & 11.6720 & 0.4948 & 0.7657 & 0.5999 & 0.4898 \\
23.0000 & 11.7420 & 0.5038 & 0.7784 & 0.6082 & 0.4977 \\
24.0000 & 11.8090 & 0.5125 & 0.7907 & 0.6163 & 0.5054 \\
25.0000 & 11.8740 & 0.5208 & 0.8027 & 0.6242 & 0.5129 \\
26.0000 & 11.9360 & 0.5290 & 0.8143 & 0.6317 & 0.5202 \\
27.0000 & 11.9970 & 0.5368 & 0.8258 & 0.6391 & 0.5274 \\
28.0000 & 12.0550 & 0.5444 & 0.8369 & 0.6463 & 0.5343 \\
29.0000 & 12.1120 & 0.5517 & 0.8479 & 0.6533 & 0.5412 \\
30.0000 & 12.1660 & 0.5587 & 0.8584 & 0.6600 & 0.5478 \\
31.0000 & 12.2200 & 0.5657 & 0.8689 & 0.6667 & 0.5544 \\
32.0000 & 12.2720 & 0.5723 & 0.8792 & 0.6732 & 0.5609 \\
\hline & & & & &
\end{tabular}

\section{Result}

Refrigerants are the working fluids in refrigeration, airconditioning, and heat-pumping systems. Accurate and comprehensive thermodynamic properties of refrigerants such as the thermal pressure coefficients are in demand by both producers and users of the materials. However, the database for the thermal pressure coefficients is small at present. Furthermore, the measurements of the thermal pressure coefficients made by different researchers often reveal systematic differences between their estimates. The researchers have led us to try to establish a correlation function for the accurate calculation of the thermal pressure coefficients for different fluids over a wide temperature and pressure ranges. The most straightforward way to derive the thermal pressure coefficient is the calculation of the thermal pressure coefficient with the use of the principle of corresponding states which covers wide temperature and pressure ranges. The principle of corresponding states calls for reducing the thermal pressure at a given reduced temperature and density to be the same for all fluids. The leading term of this correlation function is the thermal pressure coefficient of perfect gas, which each gas obeys in the low density range $[31,32]$. 
TABLE 7: Comparison of $(\partial p / \partial T)_{\rho}$ among the calculated and experimental values for R13 at $320 \mathrm{~K}[1,6,18,30]$.

\begin{tabular}{lccccc}
\hline \multirow{2}{*}{$p / \mathrm{MPa}$} & $\rho / \mathrm{mol} \cdot \mathrm{L}^{-1}$ & \multicolumn{4}{c}{$(\partial p / \partial T)_{\rho}\left(\mathrm{MPa} \cdot \mathrm{K}^{-1}\right)$} \\
& & $\mathrm{TPC}^{\text {Helmholtz }}$ & $\mathrm{TPC}^{(1)}$ & $\mathrm{TPC}^{(2)}$ & $\mathrm{TPC}^{(3)}$ \\
\hline 15.0000 & 10.1650 & 0.3269 & 0.5262 & 0.4304 & 0.3063 \\
16.0000 & 10.3040 & 0.3400 & 0.5457 & 0.4444 & 0.3175 \\
17.0000 & 10.4310 & 0.3523 & 0.5640 & 0.4574 & 0.3281 \\
18.0000 & 10.5490 & 0.3640 & 0.5813 & 0.4696 & 0.3381 \\
19.0000 & 10.6600 & 0.3751 & 0.5980 & 0.4813 & 0.3479 \\
20.0000 & 10.7630 & 0.3857 & 0.6138 & 0.4922 & 0.3571 \\
21.0000 & 10.8610 & 0.3959 & 0.6290 & 0.5028 & 0.3660 \\
22.0000 & 10.9530 & 0.4056 & 0.6436 & 0.5128 & 0.3746 \\
23.0000 & 11.0410 & 0.4149 & 0.6578 & 0.5224 & 0.3829 \\
24.0000 & 11.1240 & 0.4239 & 0.6713 & 0.5316 & 0.3909 \\
25.0000 & 11.2040 & 0.4326 & 0.6846 & 0.5406 & 0.3988 \\
26.0000 & 11.2810 & 0.4409 & 0.6975 & 0.5492 & 0.4064 \\
27.0000 & 11.3540 & 0.4490 & 0.7099 & 0.5575 & 0.4138 \\
28.0000 & 11.4250 & 0.4568 & 0.7221 & 0.5656 & 0.4211 \\
29.0000 & 11.4930 & 0.4643 & 0.7339 & 0.5734 & 0.4282 \\
30.0000 & 11.5590 & 0.4716 & 0.7455 & 0.5811 & 0.4352 \\
31.0000 & 11.6220 & 0.4786 & 0.7567 & 0.5884 & 0.4419 \\
32.0000 & 11.6840 & 0.4855 & 0.7679 & 0.5957 & 0.4486 \\
33.0000 & 11.7440 & 0.4921 & 0.7788 & 0.6027 & 0.4552 \\
34.0000 & 11.8020 & 0.4986 & 0.7894 & 0.6096 & 0.4616 \\
35.0000 & 11.8580 & 0.5048 & 0.7997 & 0.6163 & 0.4679 \\
\hline & & & & &
\end{tabular}

In this paper, we drive an expression for the thermal pressure coefficient of R11, R13, R14, R22, R23, R32, R41, and R113 dense refrigerants using the linear isotherm regularity $[18$, 28]. Unlike previous models, it has been shown in this work that the thermal pressure coefficient can be obtained without employing any reduced Helmholtz energy [9]. Only $p V T$ experimental data have been used for the calculation of the thermal pressure coefficient of R11, R13, R14, R22, R23, R32, R41, and R113 refrigerants [8]. Comparison of the calculated values of the thermal pressure coefficient using the linear isotherm regularity with the values obtained experimentally shows the validity of the use of the linear isotherm regularity for studying the thermal pressure coefficient of R11, R13, R14, R22, R23, R32, R41, and R113 refrigerants. In this work, it has been shown that the temperature dependences of the intercept and slope of using linear isotherm regularity are nonlinear. This problem has led us to try to obtain the expression for the thermal pressure coefficient of R11, R13, R14, R22, R23, R32, R41, and R113 refrigerants by extending the intercept and slope of the linearity parameters versus inversion of temperature to the third order. The thermal pressure coefficients predicted from this simple model are in good agreement with experimental data. The results show the accuracy of this method is generally quit good. The resulting model predicts accurately the thermal pressure coefficients from the lower density limit at the Boyle density from triple temperature up to about double the Boyle temperature. The upper density limit appears to be reached at 1.4 times the Boyle density. These problems have led us to try to establish a function for the accurate calculation of the thermal pressure coefficients based on equation of state theory for different refrigerants.

\section{Acknowledgment}

The authors thank the Payame Noor University for financial support.

\section{References}

[1] J. D. Happell, R. M. Price, Z. Top, and P. K. Swart, "Evidence for the removal of CFC-11, CFC-12, and CFC-113 at the groundwater-surface water interface in the Everglades," Journal of Hydrology, vol. 279, no. 1-4, pp. 94-105, 2003.

[2] R. Span and W. Wagner, "Equations of state for technical applications. III. Results for polar fluids," International Journal of Thermophysics, vol. 24, no. 1, pp. 111-162, 2003.

[3] K. A. Gillis, "Thermodynamic properties of seven gaseous halogenated hydrocarbons from acoustic measurements: $\mathrm{CHClFCF}_{3}, \mathrm{CHF}_{2} \mathrm{CF}_{3}, \mathrm{CF}_{3} \mathrm{CH}_{3}, \mathrm{CHF}_{2} \mathrm{CH}_{3}, \mathrm{CF}_{3} \mathrm{CHFCHF}_{2}$, $\mathrm{CF}_{3} \mathrm{CH}_{2} \mathrm{CF}_{3}$, and $\mathrm{CHF}_{2} \mathrm{CF}_{2} \mathrm{CH}_{2} \mathrm{~F}$," International Journal of Thermophysics, vol. 18, no. 1, pp. 73-135, 1997.

[4] E. W. Lemmon and R. T. Jacobsen, "An international standard formulation for the thermodynamic properties of 1,1,1trifluoroethane (HFC-143a) for temperatures from 161 to 450 $\mathrm{K}$ and pressures to $50 \mathrm{MPa}$," Journal of Physical and Chemical Reference Data, vol. 29, p. 521, 2000.

[5] M. J. Assael, J. Millat, V. Vesovic, and W. A. Wakeham, "The Thermal conductivity of methane and tetrafluoromethane in the limit of zero density," Journal of Physical and Chemical Reference Data, vol. 19, p. 1137, 1990.

[6] J. W. Magee, S. L. Outcalt, and J. F. Ely, "Molar heat capacity $\mathrm{Cv}$, vapor pressure, and $(\mathrm{p}, \rho, \mathrm{T})$ measurements from 92 to $350 \mathrm{~K}$ at pressures to $35 \mathrm{MPa}$ and a new equation of state for chlorotrifluoromethane (R13)," International Journal of Thermophysics, vol. 21, no. 5, pp. 1097-1121, 2000.

[7] R. Dohrn and J. M. Prausnitz, "A simple perturbation term for the Carnahan-Starling equation of state," Fluid Phase Equilibria, vol. 61, no. 1-2, pp. 53-69, 1990.

[8] L. Boltzmann, Lectures on Gas Theory, University of California Press, Berkeley, Calif, USA, 1964.

[9] V. Moeini, "New regularity for internal pressure of dense fluids," Journal of Physical Chemistry B, vol. 110, no. 7, pp. 3271-3275, 2006.

[10] V. Moeini and M. Deilam, "Determination of molecular diameter by PVT," ISRN Physical Chemistry, vol. 2012, Article ID 521827, 5 pages, 2012.

[11] R. B. Stewart and T. Jacobsen, "Thermodynamic properties of argon from the triple point to $1200 \mathrm{~K}$ with pressures to 1000 MPa," Journal of Physical and Chemical Reference Data, vol. 18, p. 639, 1989.

[12] R. D. Goodwin, "Carbon monoxide thermophysical properties from 68 to $1000 \mathrm{~K}$ at pressures to $100 \mathrm{MPa}$," Journal of Physical and Chemical Reference Data, vol. 14, p. 849, 1985.

[13] R. Span and W. Wagner, "A new equation of state for carbon dioxide covering the fluid region from the triple-point temperature to $1100 \mathrm{~K}$ at pressures up to $800 \mathrm{MPa}$," Journal of Physical and Chemical Reference Data, vol. 25, p. 1509, 1996.

[14] R. T. Jacobsen, R. B. Stewart, and M. Jahangiri, "Thermodynamic properties of nitrogen from the freezing line to $2000 \mathrm{~K}$ 
at pressures to $1000 \mathrm{MPa}$, Journal of Physical and Chemical Reference Data, vol. 15, no. 2, pp. 735-909, 1986.

[15] B. A. Younglove and J. F. Ely, "Thermophysical properties of fluids. II. methane, ethane, propane, isobutane, and normal butane," Journal of Physical and Chemical Reference Data, vol. 16, p. $577,1987$.

[16] R. D. Goodwin, "Benzene thermophysical properties from 279 to $900 \mathrm{~K}$ at pressures to 1000 Bar," Journal of Physical and Chemical Reference Data, vol. 17, p. 1541, 1988.

[17] R. D. Goodwin, "Toluene thermophysical properties from 178 to $800 \mathrm{~K}$ at pressures to 1000 Bar," Journal of Physical and Chemical Reference Data, vol. 18, p. 1565, 1989.

[18] V. Moeini, F. Ashrafi, M. Karri, and H. Rahimi, "Calculation of thermal pressure coefficient of dense fluids using the linear isotherm regularity," Journal of Physics Condensed Matter, vol. 20, no. 7, Article ID 075102, 2008.

[19] V. Moeini, "Internal pressures of lithium and cesium fluids at different temperatures," Journal of Chemical and Engineering Data, vol. 55, no. 3, pp. 1093-1099, 2010.

[20] V. Moeini, "Calculation of thermal pressure coefficient of lithium fluid by pVT data," ISRN Physical Chemistry, vol. 2012, Article ID 724230, 11 pages, 2012.

[21] T. M. Reed and K. E. Gubbins, Applied Statistical Mechanics, McGraw-Hill, 1973.

[22] W. D. Weatherford, R. K. Johnston Jr., and M. L. Valtierra, "Kinematic Viscosity of Liquid Rubidium from $67^{\circ}$ to $688^{\circ} \mathrm{C}$," Journal of Chemical and Engineering Data, vol. 9, pp. 520-524, 1964.

[23] F. Roehlich, F. Tepper, and R. L. Rankin, "Surface tension of four alkali metals to $1000^{\circ} \mathrm{C}$," Journal of Chemical and Engineering Data, vol. 13, no. 4, pp. 518-521, 1968.

[24] K. Mutsuda, K. Tamura, and M. Inui, "Instability of the electron gas in an expanding metal," Physical Review Letters, vol. 98, Article ID 096401, 2007.

[25] K. Matsuda, S. Naruse, K. Hayashi, K. Tamura, M. Inui, and Y. Kajihara, "Structural study of expanded fluid cesium," Journal of Physics: Conference Series, vol. 98, no. 1, Article ID 012003, 2008.

[26] K. Tamura, K. Matsuda, and M. Inui, "Structural and electronic properties of expanding fluid metals," Journal of Physics: Condensed Matter, vol. 20, Article ID 114102, 2008.

[27] V. Moeini, "Internal pressures of sodium, potassium, and rubidium fluids at different temperatures," Journal of Chemical \& Engineering Data, vol. 55, no. 12, pp. 5673-5680, 2010.

[28] G. Parsafar and E. A. Mason, "Linear isotherms for dense fluids: a new regularity," Journal of Physical Chemistry, vol. 97, no. 35, pp. 9048-9053, 1993.

[29] G. Parsafar, V. Moeini, and G. Najafi, "Pressure dependency of liquid vapor pressure: gibbs prediction improvement," Iranian Journal of Chemistry \& Chemical Engineering, vol. 20, pp. 3743, 2001.

[30] http://webbook.nist.gov/chemistry/.

[31] J. O. Hirschfelder, C. F. Curtiss, and R. B. Bird, Molecular Theory of Gases and Liquids, John Wiley \& Sons, New York, NY, USA, 2nd edition, 1964.

[32] Y. Ghayeb, B. Najafi, V. Moeini, and G. Parsafar, "Calculation of the viscosity of supercritical fluids based on the modified Enskog theory," High Temperatures-High Pressures, vol. 35-36, no. 2, pp. 217-226, 2003. 

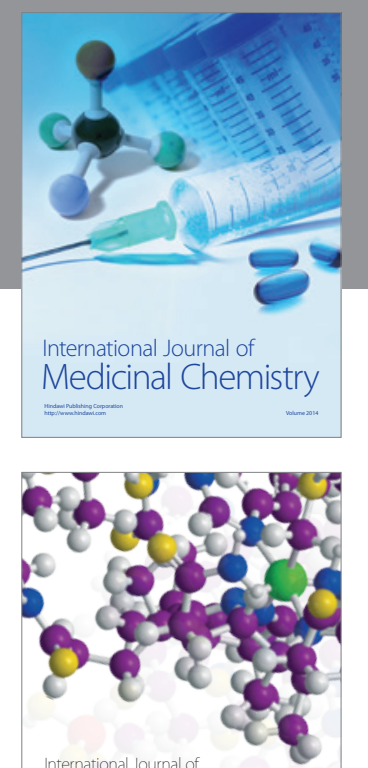

\section{Carbohydrate} Chemistry

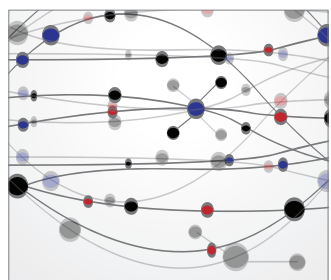

The Scientific World Journal
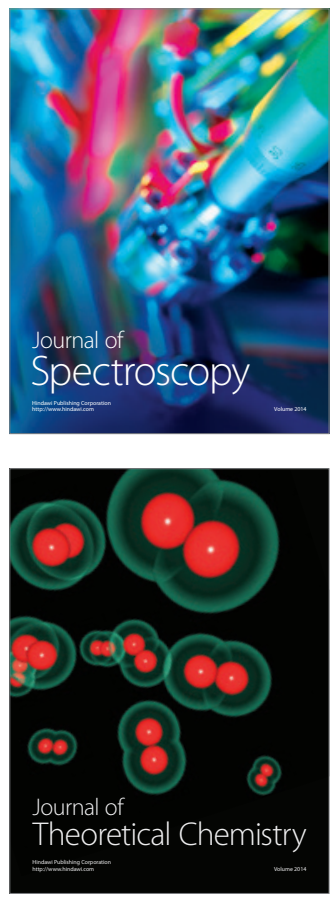
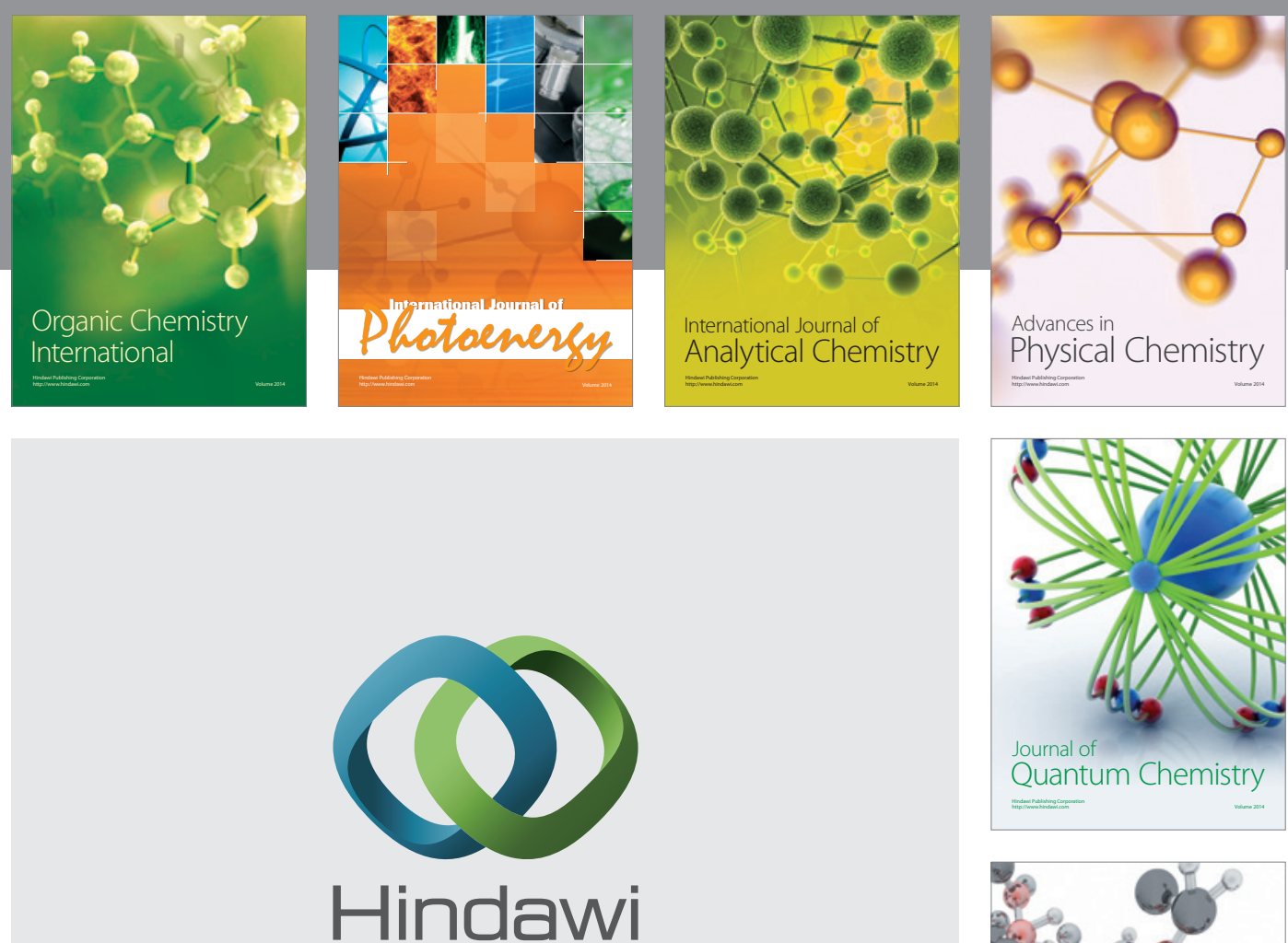

Submit your manuscripts at

http://www.hindawi.com

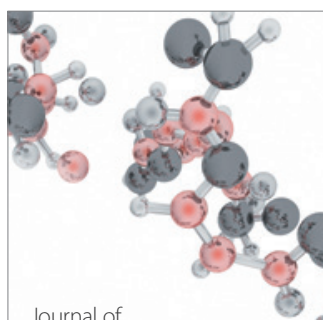

Analytical Methods

in Chemistry

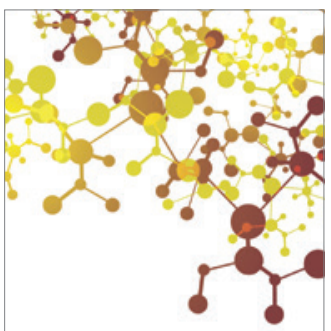

Journal of

Applied Chemistry

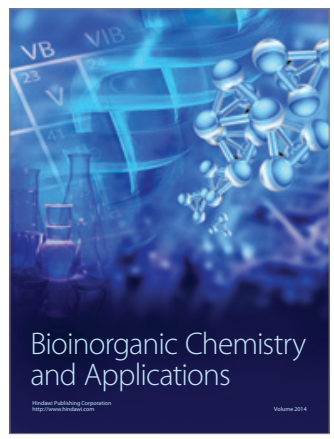

Inorganic Chemistry
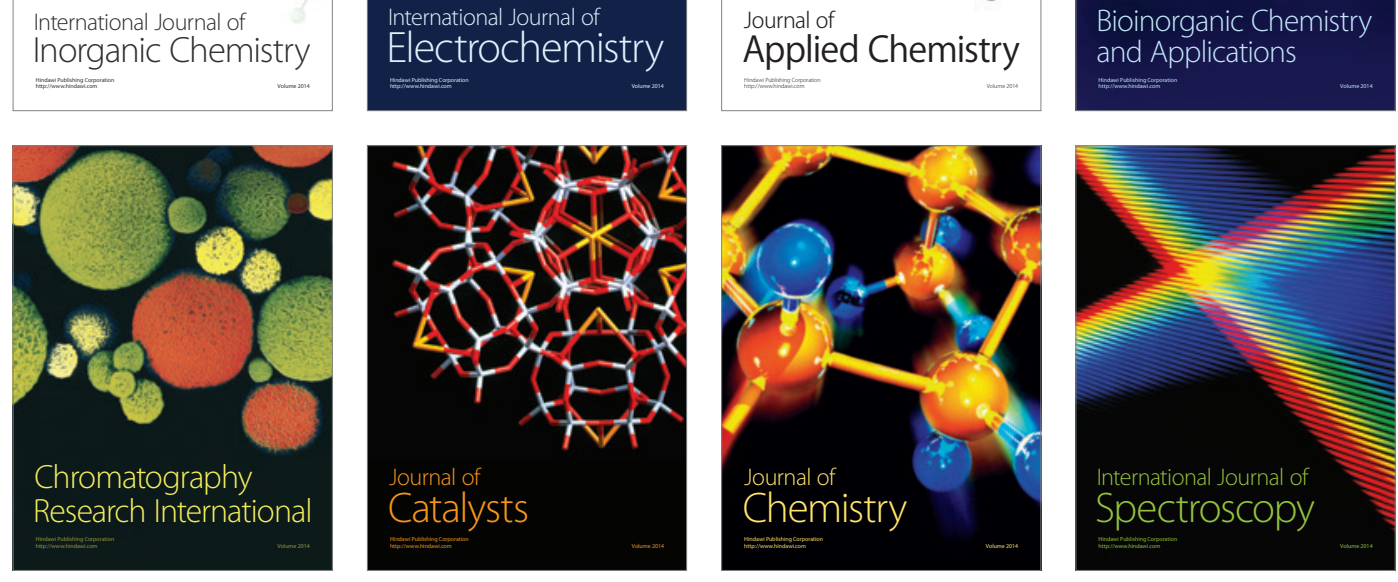